\title{
Archaeonautica
}

Archaeonautica

L'archéologie maritime et navale de la préhistoire à

l'époque contemporaine

$20 \mid 2018$

De re navali : Pérégrinations nautiques entre

Méditerranée et océan Indien

\section{Le «bateau-boomerang » prédynastique et la barque divine pharaonique}

The predynastic "boomerang boat" and the divine pharaonic boat

Lucien Basch

\section{OpenEdition}

Journals

Édition électronique

URL : http://journals.openedition.org/archaeonautica/329

DOI : 10.4000/archaeonautica.329

ISSN : 2117-6973

Éditeur

CNRS Éditions

Édition imprimée

Date de publication : 6 décembre 2018

Pagination : 143-149

ISBN : 978-2-271-12263-6

ISSN : 0154-1854

Référence électronique

Lucien Basch, « Le «bateau-boomerang » prédynastique et la barque divine pharaonique »,

Archaeonautica [En ligne], 20 | 2018, mis en ligne le 30 avril 2020, consulté le 30 avril 2020. URL :

http://journals.openedition.org/archaeonautica/329 ; DOI : https://doi.org/10.4000/archaeonautica. 329 


\title{
LE « BATEAU-BOOMERANG » PRÉDYNASTIQUE ET LA BARQUE DIVINE PHARAONIQUE
}

\author{
Lucien BASCH
}

\begin{abstract}
Résumé
Des centaines de gravures rupestres sur les rives du Nil, en HauteÉgypte et en Nubie, représentent de très nombreux bateaux. Ces gravures ne firent l'objet de recherches systématiques qu'au début des années 1930 puis, en prévision de la mise en eau du lac Nasser, les recherches internationales promues par l'UNESCO permirent la documentation systématique de ces gravures promises à une submersion définitive. Ainsi, un grand corpus d'iconographie navale d'époque prédynastique, allant du Ve millénaire jusqu'à la $\mathrm{I}^{\mathrm{re}}$ dynastie (vers 3100), fut sauvegardé et rendu disponible aux chercheurs.

Dans cet article, c'est le bateau du début du Ve millénaire dit «bateau-boomerang " qui sera analysé et comparé avec d'autres documents iconographiques afin d'en cerner les caractéristiques et en suivre les évolutions postérieures. L'hypothèse d'une identification avec une représentation d'une barque divine sera aussi discutée.
\end{abstract}

\section{Mots-clés}

Barque divine, Bateau-boomerang, Haute-Égypte, Époque prédynastique, Iconographie, Nil, Nubie
THE PREDYNASTIC "BOOMERANG BOAT" AND the DIVINE PHARAONIC BOAT

\section{Abstract}

Numerous boats are represented on hundreds of wall engravings along the banks of the Nile in Upper Egypt and Nubia. These engravings only became an object of systematic research at the beginning of the 1930s and then, in advance of the flooding of Lake Nasser, international, research promoted by UNESCO led to the systematic documentation of these engravings before they disappeared under the waters. In this way a great corpus of nautical iconography of the Predynastic period, from the $5^{\text {th }}$ millennium until the $1^{\text {st }}$ Dynasty (circa 3100), was preserved and made available to scholars.

In this article we shall examine a boat from the beginning of the $5^{\text {th }}$ millennium, the so-called "boomerang boat", and compare it with other iconographic documents in order to define the characteristics and follow later evolutions. The theory of possible identification with a representation of a divine barque will also be discussed.

Key words

Divine barque, Boomerang boat, Upper Egypt, Predynastic period, Iconography, Nile, Nubia,
Enfonçons une porte ouverte : aucune civilisation n'a été aussi liée étroitement à un seul fleuve que l'Égypte, qui doit au Nil sa naissance, son développement et plus prosaïquement, la subsistance de ses riverains, soit indirectement, par l'agriculture florissante grâce à ses alluvions, soit directement, par la pêche. Celle-ci fut pratiquée dès que les premiers nomades s'approchèrent des rives du fleuve, ce qui rend les débuts de cette activité pratiquement indatable. Cette pêche n'exigeait pas, au stade primitif, de s'aventurer sur l'eau ; de la rive, il était possible de capturer des poissons grâce à l'hameçon, à des pièges, au filet et au harpon, du moins dans les basses eaux du Nil.

Dès la sédentarisation de ces nomades, entre 10000 et 7000 ans environ avant notre ère, des vestiges d'habitats révèlent l'importance de la pêche pour l'alimentation locale, surtout en protéines. Cette pêche «primitive » se développa, comme le démontrent des restes de poissons ne vivant que dans le chenal principal (le plus profond) du Nil, ce qui a dû nécessiter de s'éloigner de la rive sur de petits radeaux ${ }^{1}$. Si des tiges de papyrus assemblées sont un moyen de transport pour un

1. À Makhadma, en Haute-Égypte, ont été découverts des poissons fumés et étêtés, stockés, ce qui indique évidemment un système économique évolué (autour de 6000 avant notre ère) (Midant-Reynes 2003, p. 44). Une navigation maritime en Mer Rouge a-t-elle existé au début du VI ${ }^{\mathrm{e}}$ millénaire ? Des restes de capridés domestiques découverts près de Ras Muhammed, sur la côte égyptienne de la Mer Rouge, pourraient témoigner d'un trafic d'animaux au départ d'Akaba (Wengrow 2006, p. 25). Si cette découverte est confirmée, je ne vois guère que des radeaux propulsés par des pagayeurs pour assurer ce transport. individu (ou un petit nombre de ceux-ci), on ne peut qualifier du terme « bateau », au sens propre du terme, ces embarcations qui flottent uniquement grâce à la nature du matériau dont elles sont composées, donc de simples flotteurs.

De vrais bateaux existaient cependant déjà vers 5000 av. J.-C. comme en témoigne un «modèle » de cette époque, au Fayoum $^{2}$, mais les modèles de la période prédynastique sont fort sommaires, variés et surtout sporadiques, ce qui décourage toute tentative de tracer d'après eux seuls une ligne d'évolution de ces barques. Or il existe une source immense d'informations, constituées par les centaines de gravures rupestres sur les rives du Nil, en Haute-Égypte et en Nubie, qui représentent, notamment, de très nombreux bateaux. Notées par plusieurs voyageurs traversant l'Égypte ${ }^{3}$, ces gravures ne firent l'objet de recherches systématiques qu'au début des années 1930 par l'archéologue autrichien H. A. Winkler ${ }^{4}$. En prévision de la mise en eau du lac Nasser, l'UNESCO lança un appel en vue de recherches internationales systématiques qui eurent pour heureux résultats la récolte de centaines de gravures promises à une submersion définitive : une manne

2. Un modèle de conteneur de grains long de $52 \mathrm{~cm}$, en forme de vraie barque à proue effilée et poupe ronde (British Museum, ${ }^{\circ}$ EA 58701, Spencer 1993, p. 21, fig. 7).

3. F. Cailliaud fut le premier à les signaler, en 1821, suivi par Ampère en 1848, Lepsius en 1852 et Linant de Bellefonds en 1868.

4. Pour l'histoire des publications voir Winkler 1938, p. 7 et 8, Huyge 1995, p. 40-44 et Morrow, Morrow 2002, p. 234. 
A

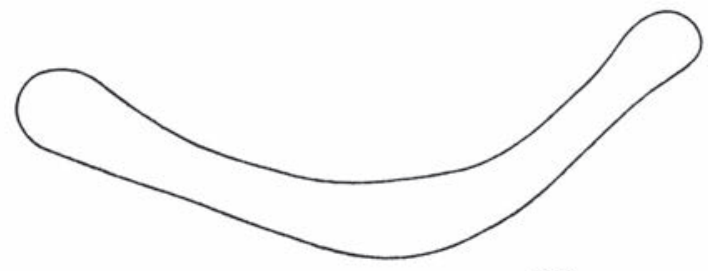

B

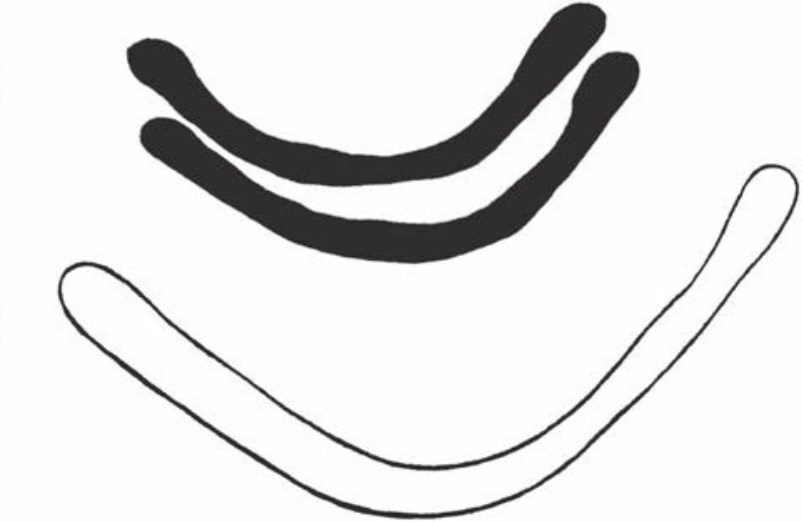

Fig. 1 : Ensemble de gravures rupestres représentant un " bateauboomerang»: A. El Kab (d'après Basch 1987) ; B. Wadi Baramiya (Morrow, Morrow 2002, p. 153-154) ; C. El Kab (Huyge 1995, II, fig. 156).

d'informations iconographiques, surtout pour les chercheurs intéressés par l'époque prédynastique, soit du V Ve millénaire (au

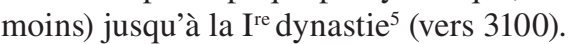

Le nombre de types de bateaux différents était tel qu'une classification s'imposait, tâche entreprise, avant tout sur la base des formes des embarcations, par plusieurs chercheurs, parmi lesquels il faut signaler Engelmayer et Červiček. Le premier aboutit à une distinction de 12 types (Engelmayer 1965), le second de 33 (Červiček 1974 ; 1986). Cependant, un accord quasi-général existe quant à l'image de la plus ancienne embarcation, très commune, remontant probablement au début du $V^{e}$ millénaire. Il s'agit d'un bateau courbe qui, de profil, ressemble à un boomerang dont les extrémités sont épaissies (fig. 1). Cette vue de profil est confirmée par un modèle en terre cuite du Musée Égyptien de Berlin, dont les extrémités, vues cette fois en plan, sont totalement aplaties ${ }^{6}$ (fig. 2).

Quel était le matériau de construction utilisé pour la très petite embarcation que représente le modèle de Berlin, très probablement l'un des plus anciens de la série ? Il faut tout d'abord exclure le papyrus et toutes espèces de joncs : l'extrémité des tiges coupées se termine toujours, sur un tel bateau, par une espèce de bouquet serré qui, en aucun cas, ne saurait présenter une forme ronde, que ce soit sur le lac Tchad ou le lac Titicaca.

Ce bateau serait-il fait d'écorce, comme les formes de bien des barques du Canada y inviteraient ? Cette écorce-là ne provient que d'arbres susceptibles de fournir de grands pans d'écorce, tels le bouleau, mais en Égypte la paléobotanique interdit de croire à leur existence, dans l'état actuel de nos connaissances.

5. L'activité des auteurs de gravures rupestres s'est poursuivie, après cette date, sans discontinuité jusqu'à nos jours : voir, par exemple, l'image d'un camion (Morrow, Morrow 2002, p. 241).

6. Ce modèle est loin d'être unique : voir les trois modèles, d'une facture beaucoup plus fruste, provenant de Nagada (Asholean Museum, Oxford 1895, 777 à 779 , Basch 1987, p. 58, fig. 95)

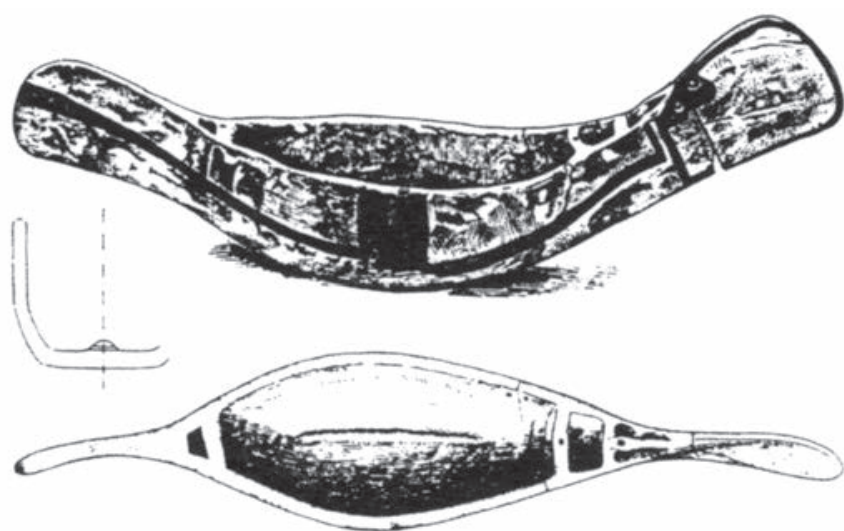

Fig. 2 : Modèle en terre cuite. Musée Égyptien, Berlin. Probablement $V^{*}$ millénaire.

Il reste le bateau de peau, matériau exceptionnellement employé pour remplacer l'écorce, qui n'est mentionné ici qu'à titre théorique 7 .

Il reste enfin le bois, débité en fragments au moyen d'outils de silex ou d'obsidienne, assemblés par des ligatures. Ce procédé est, ou a été, fréquent en Afrique, notamment au Cameroun, sur le lac Victoria (Basch 1987, p. 46, fig. 68-69) et sur le Moyen Niger (Pitot, Daget 1948, passim) ainsi que, à l'époque prédynastique, à Hiérakonpolis (Basch 1987, p. 46, fig. 67). Toutefois, l'hypothèse d'une telle construction en bois pour le modèle du Musée de Berlin me semble incompatible avec la forme de ses extrémités, faites de deux couches d'une matière non identifiée, couches « collées » l'une à l'autre sans que l'on puisse en comprendre la raison. Sur ce point le modèle demeure énigmatique. Certaines gravures rupestres montrent le renflement des deux extrémités d'une manière très accentuée, peut-être même excessive (fig. 3). On note sur cette dernière gravure l'adjonction sur l'étrave d'une tête d'animal à cornes, probablement un taureau, emblème exprimant la puissance qui deviendra plus tard celui des pharaons ${ }^{8}$.

Dans le domaine des modèles, on note, à une époque très postérieure à celui du Musée de Berlin, un «assagissement » des extrémités, qui aboutit à la forme d'une élégante pirogue, cette fois nécessairement formée de pièces de bois assemblées ${ }^{9}$ (fig. 4).

Les peintures de vases de l'époque de Nagada, vers 36003500 , ont très souvent pour sujet un bateau révélant un stade d'évolution très avancé du « bateau-boomerang ». Les extrémités sont toujours arrondies et la forme générale reste courbe (fig. 5 et 6), tandis que les avirons sont très nombreux et répartis

7. Il est fait allusion ici à une technique employée par les Indiens Malécites du Nouveau Brunswick : ils couvraient, normalement, d'écorce une carcasse d'osier, mais aussi, en cas d'urgence, de peaux d'élan (Adney, Chapelle 1964, p. 219 ; Roberts, Shackleton 1988, p. 150). En ce qui concerne l'obtention de peaux, elle ne devait poser aucun problème : le climat de l'Égypte à l'époque des premiers « bateaux-boomerang » n'était pas celui d'un désert aride, mais celui d'une savane. On rencontre dans le répertoire des plus anciennes gravures rupestres, outre des girafes et des éléphants, surtout d'innombrables bovidés.

8. Ce cas d'adjonction sur l'étrave est exceptionnel, mais non la représentation de cornes jaillissant d'une extrémité de la coque elle-même (Engelmayer 1965, pl. VI, 1 ; XII, 1 et 8a), de façon à assimiler le « bateau-boomerang » tout entier à un animal à cornes; des gravures rupestres d'un autre type de bateau prédynastique montrent une tête d'animal à cornes au sommet d'une étrave haute (sur ce sujet : Berger 1992).

9. Reconstitution très plausible de B. Landström 1970, p. 20-21, fig. 57. 


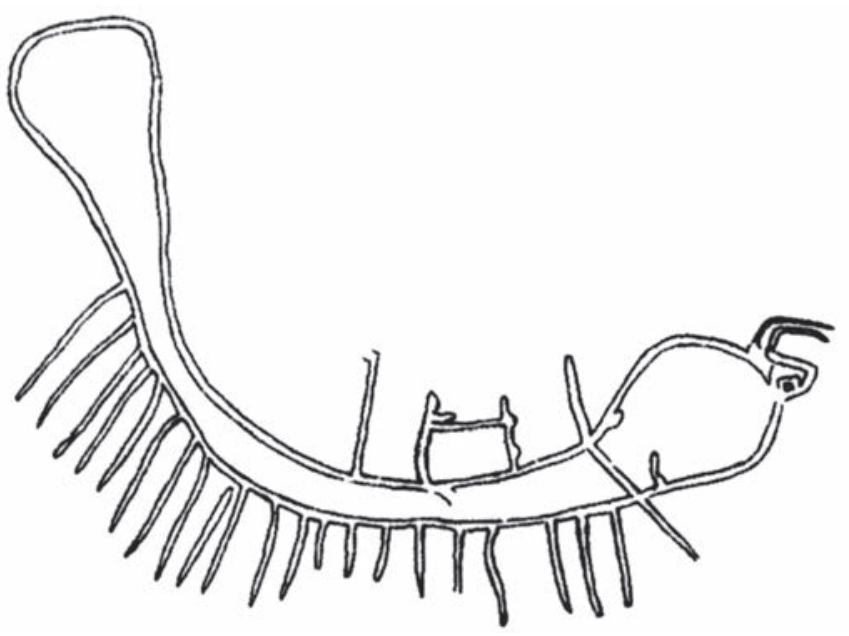

Fig. 3 : District de Sayala (Engelmayer 1965, pl. LIV, 1).

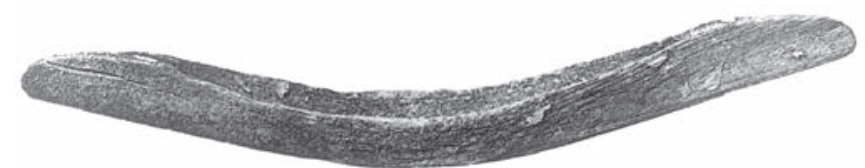

Fig. 4 : Modèle en bois. Musée Égyptien, Berlin, vers 3500 (Reisner 1913, p. $20 n^{\circ}$ 4814).

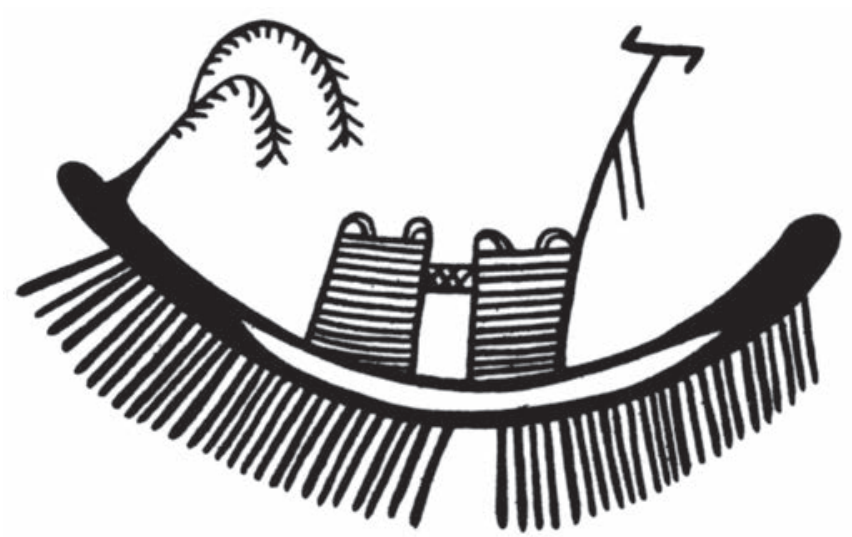

Fig. 5 : Vase nagadien, vers 3500 (Kantor 1944, pl. 3).

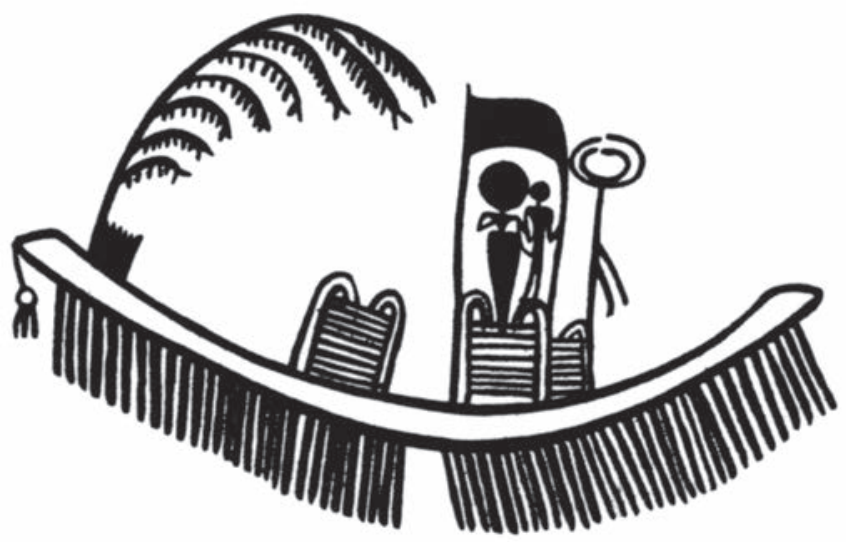

Fig. 6 : Vase nagadien, vers 3500 (Kantor 1944, pl. 3). en deux groupes séparés, de part et d'autre de deux cabines, parfois unies par une passerelle (fig. 5). Le sommet des cabines est régulièrement pourvu d'anneaux (fig. 5, flèches). Dans quelques cas, la cabine proche de la poupe abrite un personnage dont la taille considérable ne peut qu'indiquer un caractère divin (fig. 6).

En 1897 fut découverte à Hiérakonpolis, ville du Faucon et siège, au IV ${ }^{\mathrm{e}}$ millénaire, d'un royaume régional, la tombe bien connue sous le nom de «tombe 100 », ou « tombe décorée » qui contenait une fresque ${ }^{10}$ dont le sujet principal est, au sommet, une flotte de six bateaux dont cinq sont clairement les descendants directs du type du «bateau-boomerang $»^{11}$ (fig. 7). La forme de la coque rappelle celle du modèle du Musée du Caire $n^{\circ} 4814$ (fig. 4). Les autres éléments, figurés le plus distinctement sur le plus grand bâtiment (fig. 8), sont la paire de cabines reliées par une passerelle et dont l'une, à l'arrière, est surmontée par un personnage (privilège du plus grand bateau seulement). Les anneaux usuels (fig. 5) sont en place. L'éminence du personnage n'est pas due uniquement à sa place, la plus haute dans la composition, ni à sa majestueuse solitude (à l'exception d'un serviteur), mais aussi au fait qu'il occupe l'emplacement exact occupé avant lui par une divinité (fig. 6 et 9). Il est abrité par un baldaquin surmonté d'un dais arrondi et, à ses côtés, trois traits horizontaux superposés indiquent soit des armes, peut-être des javelines, soit des harpons (fig. 9).

Tout autour de la flotte, l'on voit, parmi des troupeaux, des scènes de chasse et de combats. L'une de ces scènes montre un personnage massacrant à coups de massue trois individus agenouillés, scène absolument caractéristique de l'iconographie royale à l'époque historique.

Les bateaux sont dépourvus de tout moyen de propulsion et semblent flotter magiquement dans leur empyrée, dominés par leur chef et dominant l'univers humain et animal : le grand bateau est celui d'un roitelet, très probablement le souverain de l'état d'Horus, Hiérakonpolis, voisin des cités-États de Nagada et de This/Abydos.

Il est temps de s'arrêter ici sur une caractéristique commune aux bateaux A, C, D et F : entre les deux cabines s'étend, à mi-coque, un carré sombre déjà présent sur le modèle du Musée de Berlin (fig. 2). Cette même zone est toujours dépourvue de pagayeurs (fig. 5 et 6). Ceci semble indiquer une faiblesse au centre du bateau. Or sur les barques du Moyen Niger les bateaux étaient construits en deux parties égales et identiques jointes au milieu de l'embarcation par des ligatures fortement renforcées (Pitot, Daget 1948, p. 10 et 11) (fig. 10).

Il me semble que ces particularités expliquent la «zone sombre » des barques A, C, D et F (fig. 7) : elle pourrait représenter des peaux fixées à un endroit sensible de la coque en vue d'en augmenter l'étanchéité.

Le baldaquin du bateau royal mérite de retenir l'attention. Déjà présent sur la fig. 6, document antérieur à la tombe 100 d'Hiérakonpolis où il abrite une divinité, il l'est aussi sur le manche d'ivoire du couteau dit de Gebel-el-Arak, au Musée du Louvre $^{12}$ (fig. 11) où, sous une forme anguleuse, que nous

10. La bibliographie relative à cette tombe suffit à remplir plusieurs volumes. Citons ici : Quibell 1900, Vandier 1955, p. 561-570, Case, Payne 1962, Cialowicz 1998. La tombe est datée de 3300 environ.

11. Le sixième bateau, très différent (fig. 7, E), a fait l'objet de commentaires nombreux et contradictoires, étrangers au présent sujet.

12. Musée du Louvre, $\mathrm{n}^{\circ}$ E11517, vers 3450. Voir notamment : Vandier, 1952, p. 531-539, Delange 2009 (très belles photographies). 


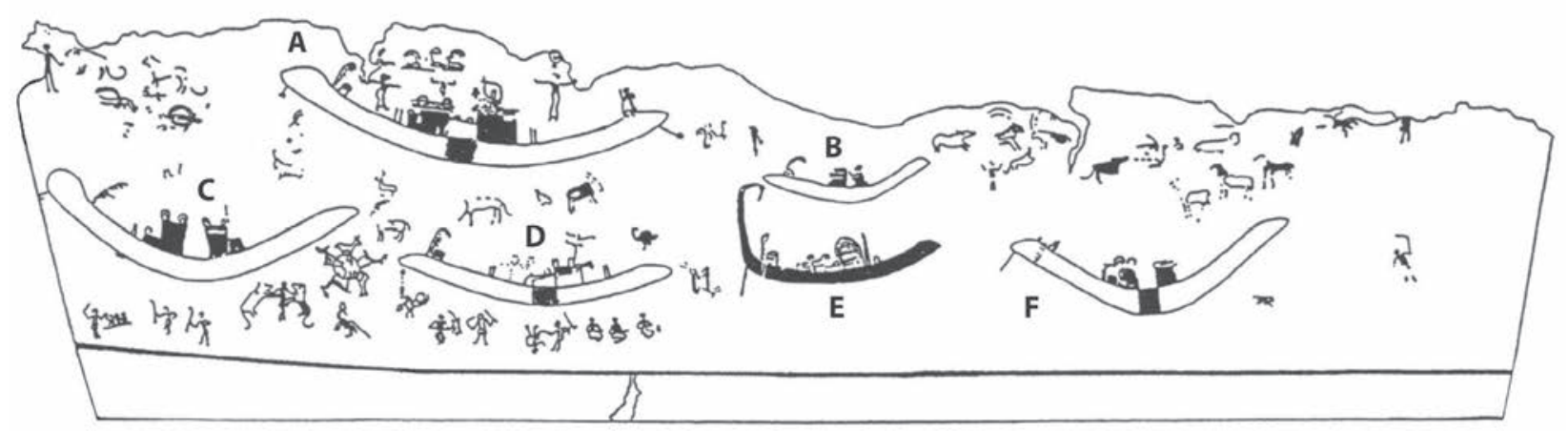

Fig. 7 : Ensemble de la fresque de la « tombe 100 », Hiérakonpolis vers 3300 (Quibell, Green 1902, pl. LXXV).

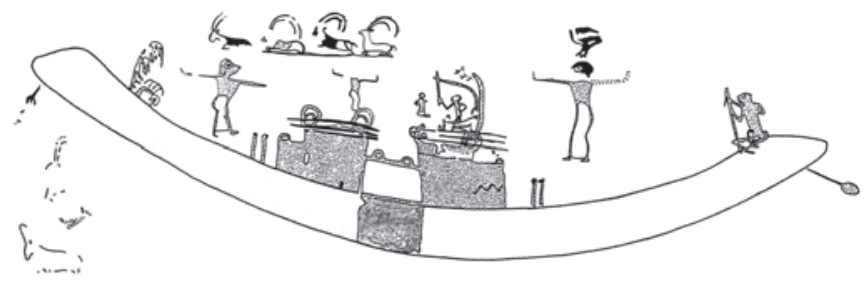

Fig. 8 : Détail de la fig. 7 : la grande barque (Spencer 1993, p. 36-37).

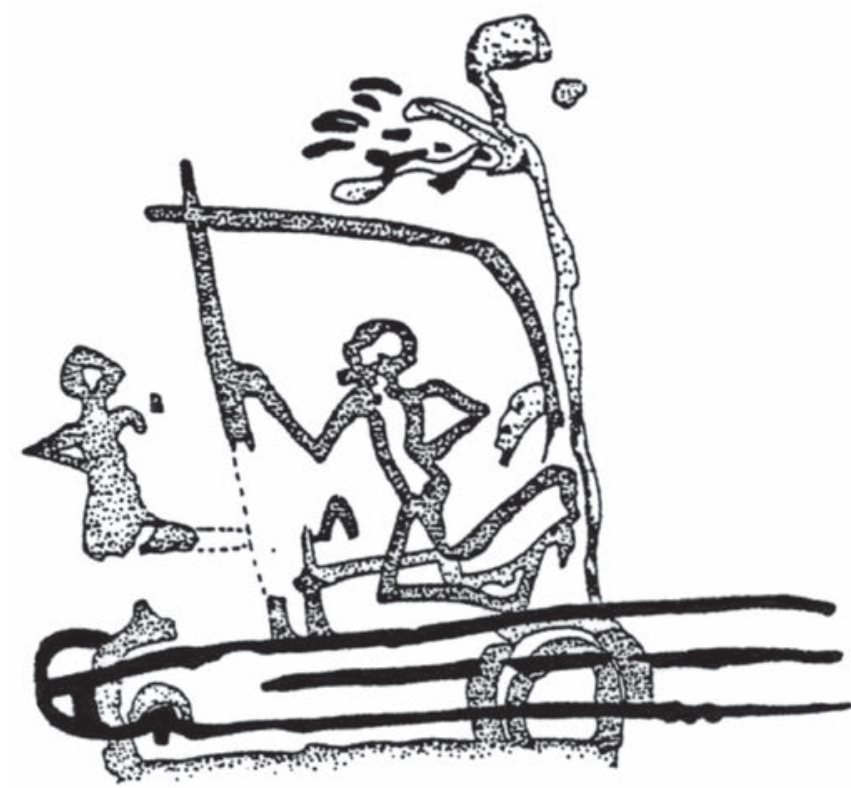

Fig. 9 : Détail de la fig. 7 : la cabine arrière (Cialowicz 1998).

retrouverons bientôt, il joue le rôle de la cabine arrière, reliée par une passerelle à celle de l'avant.

La lignée du «bateau-boomerang » se poursuit, et pratiquement se termine, par une gravure rupestre exceptionnelle, photographiée par H.A. Winkler dans le Wadi Arab (fig. 12) et publiée par H. Kees ${ }^{13}$.

13. Kees 1961, fig. 3a. Le Wadi Abad (ou Wadi Abbad), aussi appelé Wadi Mia, débouche dans la vallée en face d'Edfou. H. Kees, le premier, à ma connaissance, à l'avoir publiée, précise bien que la photo a été prise par «H.A.
Les extrémités du bateau sont toujours arrondies en boudins, caractéristique totalement exclue à l'époque pharaonique. Ses deux cabines sont conformes aux canons prédynastiques, dont leur réunion par une passerelle. Les pagaies sont représentées des deux bords.

La cabine proche de la poupe est surmontée d'un baldaquin, vide d'occupant et la cabine proche de la proue est surmontée d'un personnage coiffé d'une couronne blanche, caractéristique, à l'époque pharaonique tout au moins, de la royauté de la HauteÉgypte. Il porte d'une main la houlette, futur sceptre « heka » et de l'autre le fouet (encore dit « chasse-mouches »), modèle du sceptre « nekhekh », tous deux emblèmes royaux.

Avec ce document se termine en gloire la descendance peut-être devenue symbolique et privilège du roi du « bateauboomerang », à l'exception d'une image très déformée sur un peigne de Ouadjit-Djet, quatrième pharaon de la première dynastie (vers 2080) (fig. 13).

Le baldaquin royal surmonté d'un dais anguleux survit sous le règne de Narmer, premier roi de la première dynastie (vers 3100) (fig. 14), ou sous la forme d'un dais arrondi (tablette de Hor-Aha, successeur du précédent) (fig. 15).

Une autre tablette de Hor-Aha présente un intérêt majeur : on y voit une coque typique de l'époque pharaonique, aux extrémités hautes, ce qui marque une rupture totale avec le «bateau-boomerang » (fig. 16).

Au Nouvel Empire, et probablement déjà au Moyen Empire, l'architecture canonique du temple traduit toujours l'association intime du dieu et de sa barque : le logis du dieu, le Saint des Saints, était situé au fond du temple, et sa barque personnelle était abritée exactement devant sa demeure, dans le Sanctuaire de la Barque. J. Vandier écrivait à ce sujet : « le culte s'adressait à une statue divine ou à une barque sacrée. Ni la statue, ni la barque ne restaient continuellement dans le sanctuaire, le symbole divin était sorti en procession ${ }^{14} »$.

Winckler» $(s i c)$; or elle ne figure ni dans les ouvrages de H. A. Winkler luimême, ni dans ses archives photographiques déposées à l'Egypt Exploration Society à Londres et pour la plupart non publiées. Kees qualifie ce document de « rock painting », mais tout porte à croire qu'il s'agit d'une gravure rupestre et non d'une peinture.

14. Vandier 1955, p. 793. Il y a lieu de souligner l'importance, dans cette citation, du mot « ou », qui implique justement, dans la théologie égyptienne, la quasi-équivalence de la Statue et de la Barque. Foucart (1924) a minutieusement décrit les voyages des dieux à bord de leur barque personnelle, voyages au cours desquels ils se rendaient visite par le fleuve (abondante iconographie). La fig. 12 pourrait représenter un tel épisode à la fin de l'époque prédynastique. 


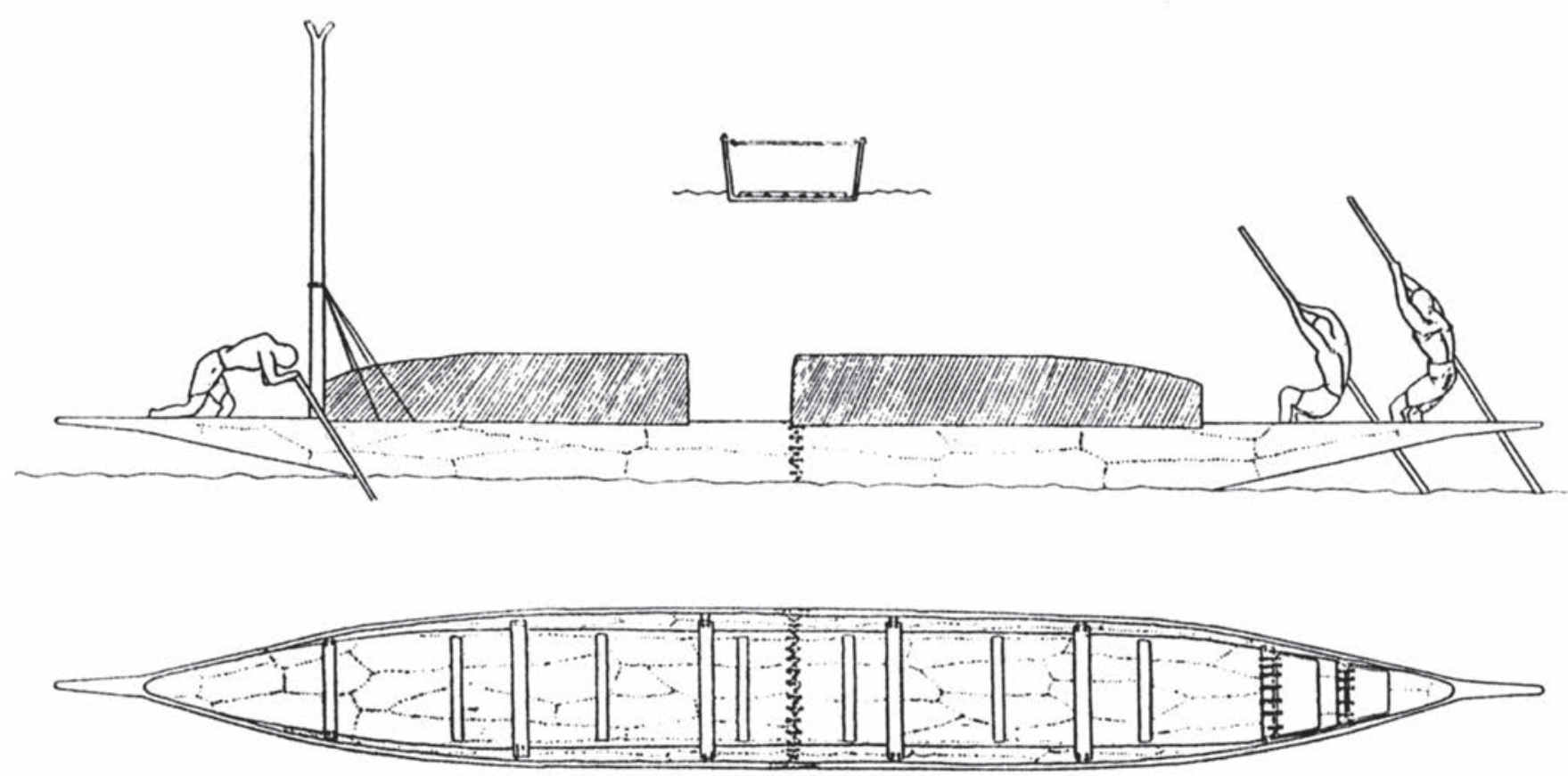

Fig. 10 : Barque de Djenné (Pitot, Daget 1948, p. 10 et 11).
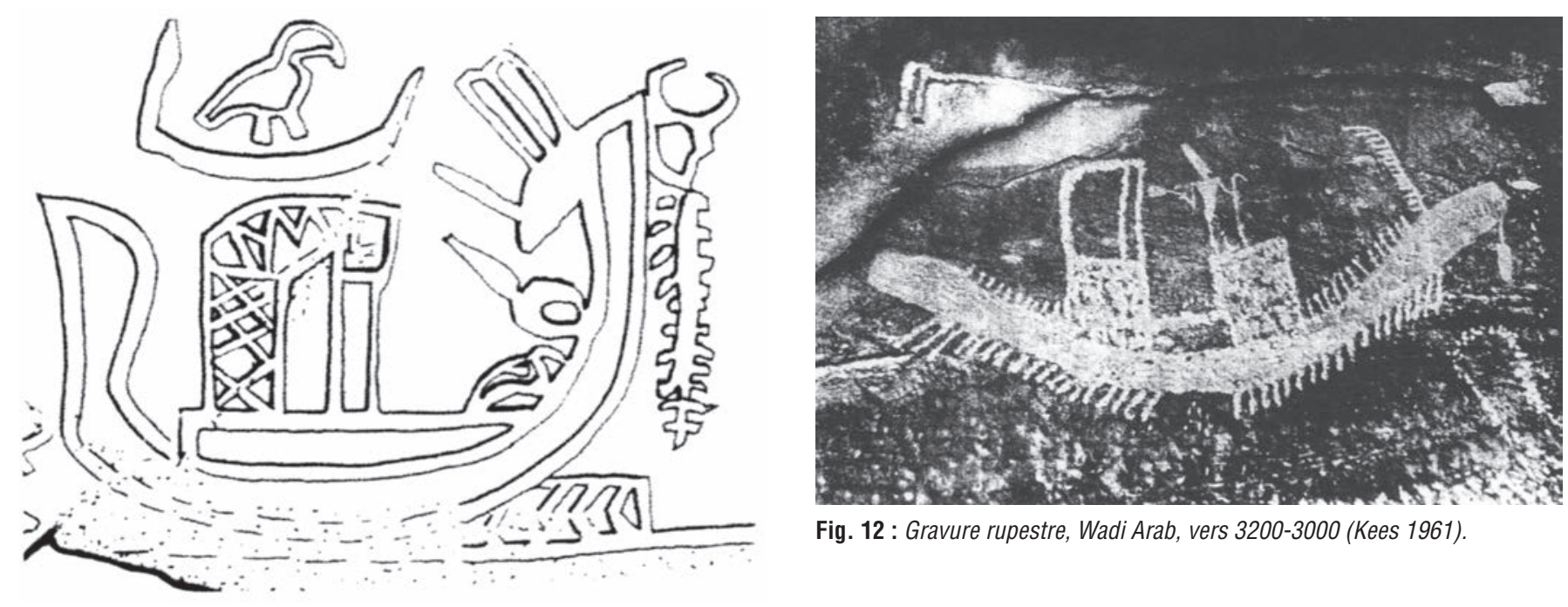

Fig. 12 : Gravure rupestre, Wadi Arab, vers 3200-3000 (Kees 1961).

Fig. 11 : Manche de couteau en ivoire, dit du Gebel el Arak, Musée du Louvre, E 11517, vers 3450 (Basch 1987, p. 60, fig. 103).

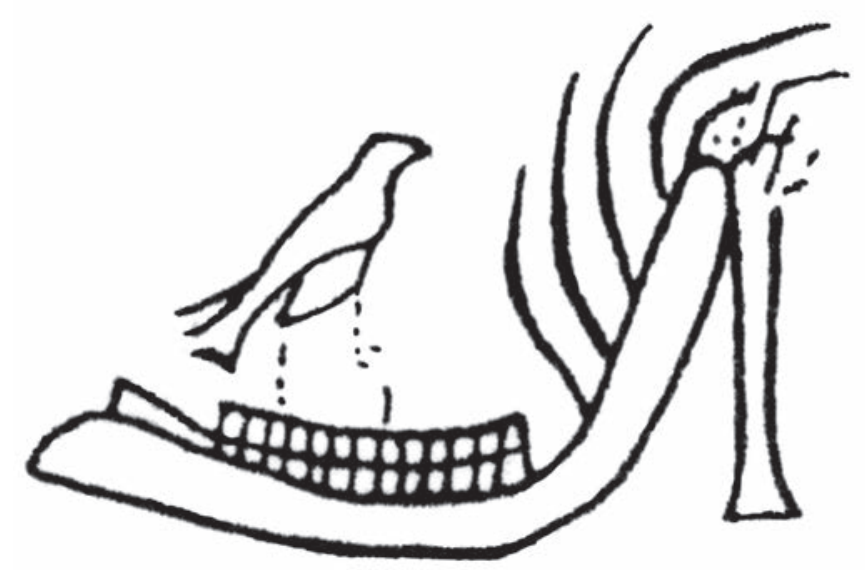

Fig. 13 : Détail d'un peigne du pharaon Oudinou, vers 2080. 


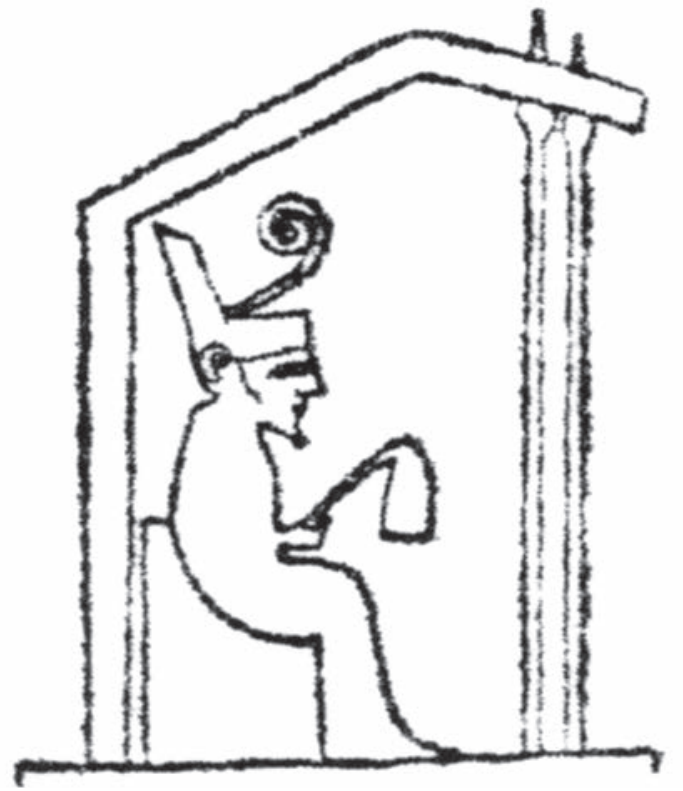

Fig. 14 : Massue de Narmer, premier pharaon de la $1^{\text {re }}$ dynastie, vers 3100. Ashmolean Museum, Oxford.

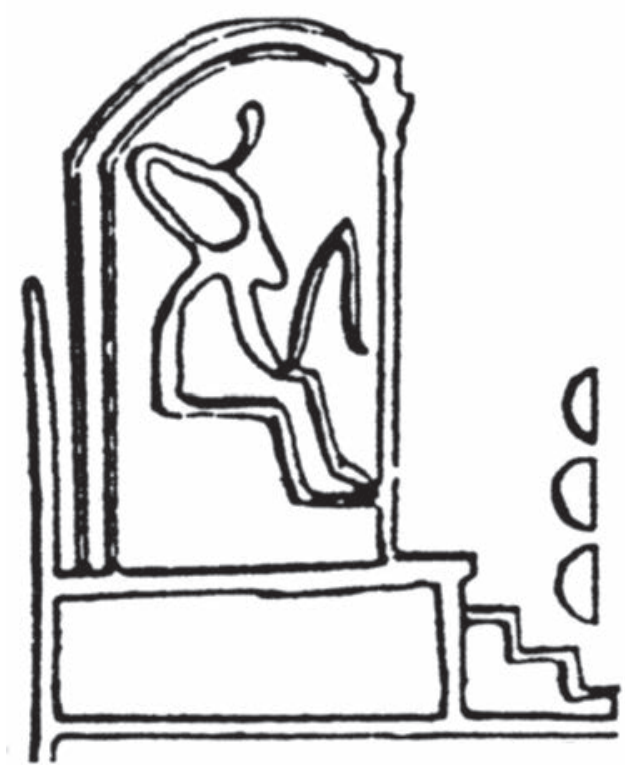

Fig. 15 : Tablette au nom du pharaon Hor-Aha, $2^{e}$ pharaon de la $1^{r e}$ dynastie, vers 3000 .

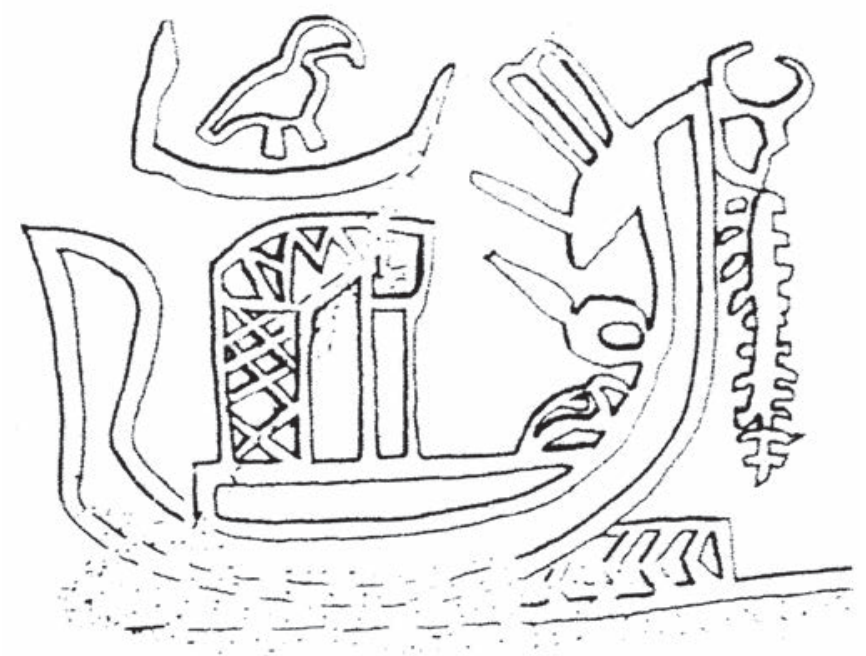

Fig. 16 : Tablette du pharaon Hor-Aha.

\section{LUCIEN BASCH}

Lucien Basch (5 février 1930-25 janvier 2018) est un juriste de formation et de profession (il fut premier Avocat général auprès de la Cour d'Appel de Bruxelles) qui s'est consacré à l'étude de l'histoire de l'architecture navale antique méditerranéenne, avec un intérêt tout particulier pour l'univers maritime de la Grèce antique. Il a également orienté ses travaux vers l'histoire de l'architecture navale de l'Égypte des époques pré- et pharaonique. Remarquable exégète des sources écrites antiques, Lucien Basch a été un tout aussi exceptionnel exégète des sources iconographiques. Ces dernières l'ont mené à écrire une histoire de l'architecture navale de l'Antiquité méditerranéenne dont son ouvrage Le musée imaginaire de la marine antique (1987), par la richesse de sa documentation et la pertinence de ses analyses, demeure et demeurera longtemps encore une référence fondamentale. 


\section{BIBLIOGRAPHIE}

\section{Adney E. T., Chapelle H.}

1964 The Bark Canoes and Skin Boats of North America, Washington, D. C., Museum of History and Technology.

BASCH L.

1987 Le musée imaginaire de la marine antique, Athènes, Institut Hellénique pour la préservation de la Tradition Nautique.

BERgER M. A.

1992 Predynastic Animal-Headed Boats from Hierakonpolis and Southern Egypt, dans Friedman R., Adams B. (eds), The Followers of Horus. Studies Dedicated to Michael Allen Hoffman, Oxford, Oxbow books (Egyptian Studies Association Publication 2, Oxbow Monograph 20), p. 107-120.

\section{Case H., Payne J. C.}

1962 Tomb 100: The Decorated Tomb at Hierakonpolis, Journal of Egyptian Archaeology, 48, p. 5-18.

\section{ČERVIČEK P.}

1974 Felsbilder des Nord-Etbai, Oberrägyptens und Unternubiens. Ergebnisse der VIII. DIAFE nach Ägypten 1926, Wiesbaden (Ergebnisse der Frobenius-Expeditionen 16).

1986 Rock Pictures of Upper Egypt and Nubia, Rome, Istituto Universitario Orientale-Napoli (Annali Suppl. 46, 1).

Cialowicz K.

1998 Once more the Hierakonpolis wall painting, dans Eyre Y. (ed.), Proceedings of the Seventh International Congress of Egyptologists, Cambridge 1995, Leuven, Peeters (Orentalia Lovaniettsa Analecta, 82), p. 273-280.

Delange E.

2009 Le poignard égyptien dit «du Gebel el-Arak», Paris, Édition du Louvre, Somogy Éditions d'Art.

\section{Engelmayer R.}

1965 Die Felsgravierungen im Distrikt Sayala-Nubien. Teil 1. Die Schiffsdarstellungen, Vienne, (Berichte des Österrreichischen Nationalkomitees der UNESCO-Aktion für die Rettung der Nubischen Altertümer 2, Denkschriften der Osterrreichische Academie der Wissenschaften, Philosophisch-historische Klasse 90).

\section{FOUCART G.}

1924 Études thébaines. La belle fête de la Vallée, BIAO, 24, p. 1-209.

HUYGE D.

1995 De Rotstekeningen van Elkab (Boven-Egypte). Registratie en Interpretatie, Thèse de doctorat, Louvain.
KANTOR H.

1944 The Final Phase of Predynastic Culture. Gerzean or Semainean (?), Journal of Near Eastern Studies, 3, p. 110-136.

KeEs H.

1961 Ancient Egypt, A Cultural Topography, Londres, Faber and Faber.

LANDSTRÖM B.

1970 Ships of the Pharaos, Londres, Allen and Unwin.

Mitant-Reynes B.

1993 Aux origines de l'Égypte. Du néolithique à l'émergence de l'État, Paris, Fayard.

Morrow M., Morrow M.

2002 Desert Rats, Rock Art, Topographical Survey in Egypt's Eastern Desert, Site Catalogue, Londres, Bloomsbury Summer School University College.

Pitot A., Daget J.

1948, Les barques du Moyen Niger, Paris, Djibouti, Centre I.F.A.N.

Quibell J. F., GReEN F. W.

1902 Hierakonpolis, II, Londres (Egyptian Research Account, 5).

REISNER G.A.

1913 Models of Ships and Boats, Catalogue général des Antiquités Égyptiennes du Musée du Caire, $n^{\circ}$ 4898-4976 et 5034-5200, Le Caire, IFAO.

Roberts K.G., SHACKLETON P.

1988 Canoë (de l'Arctique au Canada), Paris, Denoël.

SPENCER A.J.

1993 Early Egypt, The Rise of Civilisation in the Nile Valley, Londres, British Museum Press.

VANDIER J.

1955 Manuel d'archéologie égyptienne, II, Les grandes époques, 2, l'architecture religieuse et civile, Paris, Édition A. et J. Picard.

Wengrow D.

2006 The Archaeology of Early Egypt, Cambridge, Cambridge University Press.

WINKLER H.A

1937 Völkerund Völkerbewegungen in vorgeschichtlichten Oberätgypten im Lichte neuer Felsbilderfunde, Stuttgart.

1938 Rock Drawings of Southern Upper Egypt I, Londres, Egypt Exploration Fund.

1939 Rock Drawings of Southern Upper Egypt II, Londres, Egypt Exploration Fund. 